\title{
ON RADII OF CONVEXITY AND STARLIKENESS OF SOME CLASSES OF ANALYTIC FUNCTIONS
}

\author{
KHALIDA INAYAT NOOR \\ Mathematics Department \\ College of Science \\ King Saud University \\ Riyadh 11451 \\ Saudi Arabia \\ (Received December 5, 1989 and in revised form March 21, 1990)
}

\begin{abstract}
Let $P[A, B],-1 \leq B<A \leq 1$, be the class of functions $p$ such that $p(z)$ is subordinate to $\frac{1+A z}{1+B z}$. Let $P\left(\alpha_{1}\right)$ be the class of functions with positive real part greater than $\alpha_{1}, 0 \leq \alpha_{1} \leq 1$. It is clear that $P[A, B] \subset P\left(\frac{1-A}{1-B}\right) \subset P[1,-1]$. The principal results in this paper are the determination of the radius of $\beta$-starlikeness and $\beta$-convexity of $f(z)$ with $\beta=\frac{1-A}{1-B}$, when $f(z)$ is restricted to certain classes of univalent and analytic functions related with $P[A, B]$.
\end{abstract}

KEY WORDS AND PHRASES. Subordinate, starlike and convex functions, bounded boundary rotation, radius, close-to-convex functions.

1991 AMS SUBJECT CLASSIFICATION CODE. 30A32, 30A34, 30C45.

\section{INTRODUCTION.}

Let $f$ be analytic in $E=\{z:|z|<1\}$, and be given by

$$
f(z)=z+\sum_{n=2}^{\infty} a_{n} z^{n}
$$

A function $g$, analytic in $E$, is called subordinate to a function $G$ if there exists a Schwarz function $w(z)$, $w(z)$ analytic in $E$ with $w(0)=0$ and $|w(z)|<1$ in $E$, such that $g(z)=G(w(z))$.

In [1], Janowski introduced the class $P[A, b]$. For $A$ and $B,-1 \leq B<A \leq 1$, a function $p$, analytic in $E$ with $p(0)=1$ belongs to the class $P[A, B]$ if $p(z)$ is subordinate to $\frac{1+A z}{1+B z}$.

Also $C[A, B]$ and $S^{*}[A, B]$ denote the classes of functions, analytic in $E$ and given by (1.1) such that $\frac{\left(z f^{\prime}(z) y\right.}{f(z)} \varepsilon P[A, B]$ and $\frac{z f^{\prime}(z)}{f(z)} \varepsilon P[A, B]$ respectively. For $A=1$, and $B=-1$, we note that $C[1,-1] \equiv C$ and $S^{*}[1,-1] \equiv S^{*}$, the classes of convex and starlike functions in $\varepsilon$. Also $S^{*}[A, B] \subset S^{*}\left(\frac{1-A}{1-B}\right) \subset S^{*}[1,-1]$ and $C[A, B] \subset C\left(\frac{1-A}{1-B}\right) \subset C[1,-1]$, where $S^{*}\left(\frac{1-A}{1-B}\right)$ and $C\left(\frac{1-A}{1-B}\right)$ denote the classes of starlike and convex functions of order $\frac{1-A}{1-B}$ respectively. These classes were first introduced by Robertson in [2].

A function $f$, analytic in $E$ and given by (1.1), is said to be in the class $R_{k}[A, B],-1 \leq B<A \leq 1$, if and only if 
Hence

$$
\frac{\left(z f^{\prime}(z)\right)^{\prime}}{f^{\prime}(z)}-\frac{1-A}{1-B}=p(z)+\frac{z p^{\prime}(z)}{p(z)}-\frac{1-A}{1-B}
$$

Using Lemma 2.3 for $\alpha=1=\beta$, we have for $R_{1} \leq R_{2}$

$$
\begin{aligned}
\operatorname{Re}\left[\frac{\left(z f^{\prime}(z)\right)^{\prime}}{f^{\prime}(z)}-\frac{1-A}{1-B}\right] & \geq \frac{1-(3 A-B) r+A^{2} r^{2}}{(1-A r)(1-B r)}-\frac{1-A}{1-B} \\
& =\frac{A-B}{1-B}\left[\frac{1-(2+A-B) r+A r^{2}}{(1-A r)(1-B r)}\right],
\end{aligned}
$$

and this implies that $\operatorname{Re}\left[\frac{\left(z f^{\prime}(z) r\right.}{f^{\prime}(z)}-\frac{1-A}{1-B}\right] \geq 0$ for $|z|<r_{0}$, where $r_{0}$ is given by (3.1). The inequality $R_{1}<R_{2}$ is satisfied whenever $T(r)=1-(2+A-B) r+A r^{2} \geq 0$. But $T(0)=1>0$ and $T(1)=B-1<0$. So $T(r)$ has at least one root in $(0,1)$. Let $r_{0}$, given by $(3.1)$ be that root of $T(r)=0$. Then in $\left[0, r_{0}\right), R_{1}<R_{2}$ and hence $f \varepsilon C\left(\frac{1-A}{1-B}\right)$ for all $z$ with $|z|=r \leq r_{0}<1$.

This result is sharp for the function $f_{0} \varepsilon S^{*}[A, B]$ such that

$$
\frac{z f_{0}^{\prime}(z)}{f_{0}(z)}=\frac{1+A z}{1+B z}
$$

THEOREM 3.2. Let $g \varepsilon S^{*}[A, B]$ and let $\frac{z f^{\prime}(z)}{g(z)} \varepsilon P[A, B]$. Then $f \varepsilon C\left(\frac{1-A}{1-B}\right)$ for $|z|<r_{0}$, where $r_{0}$ is given by (3.1).

PROOF. $z f^{\prime}(z)=g(z) p(z), \quad \quad p \varepsilon P[A, B]$

This gives us

$$
\frac{\left(z f^{\prime}(z)\right)^{\prime}}{f^{\prime}(z)}=\frac{z g^{\prime}(z)}{g(z)}+\frac{z p^{\prime}(z)}{p(z)}
$$

Applying the usual inequalities, we obtain

$$
\begin{aligned}
\operatorname{Re}\left[\frac{\left(z f^{\prime}(z)\right)^{\prime}}{f^{\prime}(z)}-\frac{1-A}{1-B}\right] & \geq \frac{1-A r}{1-B r}-\frac{(A-B) r}{(1-A r)(1-B r)}-\frac{1-A}{1-B} \\
& =\frac{(A-B)[1-(2+A-B) r+A r]}{(1-B)(1-A r)(1-B r)}
\end{aligned}
$$

Hence we obtain the required result that $f \varepsilon C\left(\frac{1-A}{1-B}\right)$ for $|z|<r_{0}$ and $r_{0}$ is given by (3.1).

THEOREM 3.3. Let $g \varepsilon S^{*}[A, B]$ and $\frac{z f^{\prime}(z)}{g(z)} \varepsilon P[A, B]$. Then $\frac{\left(z f^{\prime}(z) y\right.}{g^{\prime}(z)} \varepsilon P\left(\frac{1-A}{1-B}\right)$ for $|z|<r_{0}$, where $r_{0}$ is given by (3.1).

PROOF. We have $z f^{\prime}(z)=g(z) p(z), p \varepsilon P[A, B]$ and so

$$
\frac{\left(z f^{\prime}(z)\right)^{\prime}}{g^{\prime}(z)}=p(z)+\frac{g(z)}{z g^{\prime}(z)} \cdot z p^{\prime}(z)
$$

Thus

$$
\begin{aligned}
\operatorname{Re}\left[\frac{\left(z f^{\prime}(z)\right)^{\prime}}{g^{\prime}(z)}-\frac{1-A}{1-B}\right] & \geq \operatorname{Re} p(z)\left[1-\frac{(1-B r)}{(1-A r)} \cdot \frac{(A-B) r}{(1-A r)(1-B r)}\right]-\frac{1-A}{1-B} \\
& \geq \frac{(1-A r)}{(1-B r)}\left[\frac{1-(3 A-B) r+A^{2} r^{2}}{(1-A r)(1-B r)}\right]-\frac{1-A}{1-B} \\
& =\frac{(A-B)}{(1-B)}\left[\frac{1-(2+A-B) r+A r^{2}}{(1-A r)(1-B r)}\right]
\end{aligned}
$$




$$
f(z)=\frac{\left(S_{1}(z)\right)^{\frac{k}{4}+\frac{1}{2}}}{\left(S_{2}(z)\right)^{\frac{k}{4}-\frac{1}{2}}}, \quad S_{1}, S_{2} \varepsilon S^{*}[A, B] .
$$

Clearly $k \geq 2$ and $R_{2}[A, B]=S^{*}[A, B]$. Also $R_{k}[1,-1]=U_{k}$, the class of functions with bounded radius rotation discussed in [3].

Similarly we can define the class $V_{k}[A, B]$ as follows. A function $f$, analytic in $E$ and given by (1.1) belongs to $V_{k}[A, B], k \geq 2$, if and only if

$$
f^{\prime}(z)=\frac{\left(S_{1}(z) / z\right)^{\frac{k}{4}+\frac{1}{2}}}{\left(S_{2}(z) / z\right)^{\frac{k}{4}-\frac{1}{2}}}, \quad S_{1}, S_{2} \varepsilon S^{*}[A, B]
$$

From (1.2) and (1.3), it is clear that

$$
f \varepsilon V_{k}[A, B] \text { if and only if } z f^{\prime} \varepsilon R_{k}[A, B]
$$

It may be noted that $V_{2}[A, B]=C[A, B]$ and $V_{k}[1,-1]=V_{k}$, the class of functions of bounded rotation first discussed by Paatero [4].

\section{PRELIMINARY RESULTS}

LEMMA 2.1 [5] Let $\mathrm{p} \varepsilon \mathrm{P}[\mathrm{A}, \mathrm{B}]$. Then

$$
\frac{1-A r}{1-B r} \leq \operatorname{Re} p(z) \leq|p(z)| \leq \frac{1+A r}{1+B r}
$$

The following is the extension of Libera's result [6].

LEMMA 2.2. Let $N$ and $D$ be analytic in $E, D$ map onto a many-sheeted starlike region. $N(0)=0=D(0)$ and $\frac{N^{\prime}(z)}{D^{\prime}(z)} \varepsilon P[A, B]$. Then $\frac{N(z)}{D(z)} \varepsilon P[A, B]$. For the proof of this result we refer to [5].

LEMMA 2.3. [7] Let $p \varepsilon P[A, B]$. Then, for $z \varepsilon E, \alpha \geq 0$ and $\beta \geq 0$, we have

$$
\operatorname{Re}\left\{\alpha p(z)+\beta \frac{z p^{\prime}(z)}{p(z)}\right\} \geq \mid \begin{array}{ll}
\frac{\alpha-\{\beta(A-B)+2 \alpha A\} r+\alpha A^{2} r^{2}}{(1-A r)(1-B r)}, & R_{1} \leq R_{2} \\
\beta \frac{A+B}{A-B}+\frac{2\left[\left(L_{1} K_{1}\right)^{1 / 2}-\beta\left(1-A B R^{2}\right)\right]}{(A-B)\left(1-r^{2}\right)}, & R_{2} \leq R_{1}
\end{array}
$$

where

$$
R_{1}=\left(\frac{L_{1}}{K_{1}}\right)^{1 / 2}, \quad R_{2}=\frac{1-A r}{1-B r}, \quad L_{1}=\beta(1-A)\left(1+\dot{A} r^{2}\right)
$$

and

$$
K_{1}=\alpha(A-B)\left(1-r^{2}\right)+\beta(1-B)\left(1+B r^{2}\right)
$$

This result is sharp.

\section{MAIN RESULTS.}

THEOREM 3.1. Let $f \varepsilon S^{*}[A, B]$. Then $f \varepsilon C\left(\frac{1-A}{1-B}\right)$ for

$$
|z|<r_{0}=\frac{2}{(2+A-B)+\sqrt{(2+A-B)^{2}-4 A}}
$$

This result is sharp.

PROOF. We have $z f^{\prime}(z)=f(z) p(z), p \varepsilon P[A, B]$ 
Hence $\frac{\left(z f^{\prime}(z)\right)}{g^{\prime}(z)} \varepsilon P\left(\frac{1-A}{1-B}\right)$ for $|z|<r_{0}$, where $r_{0}$ is given by (3.1).

Our next result is about the radius of convexity problem for the class $V_{k}[A, B]$.

THEOREM 3.4. Let $f \varepsilon V_{k}[A, B], k \geq 2$. Then $f \varepsilon C\left(\frac{1-A}{1-B}\right)$ for $|z|<r_{1}$, where

$$
r_{1}=\frac{4}{k(1-B)+\sqrt{k^{2}(1-B)^{2}+16 B}}
$$

PROOF. Since $f \varepsilon V_{k}[A, B]$, we have from (1.3)

$$
f^{\prime}(z)=\frac{\left(S_{1}(z) / z\right)^{\frac{k}{4}+\frac{1}{2}}}{\left(S_{2}(z) / z\right)^{\frac{k}{4}-\frac{1}{2}}}, \quad S_{1}, S_{2} \varepsilon S^{*}[A, B]
$$

This implies that

$$
\frac{\left(z f^{\prime}(z)\right)^{\prime}}{f^{\prime}(z)}=\left(\frac{k}{4}+\frac{1}{2}\right) p_{1}(z)-\left(\frac{k}{4}-\frac{1}{2}\right) p_{2}(z), p_{1}, p_{2} \varepsilon P[A, B]
$$

so

$$
\begin{aligned}
\operatorname{Re}\left[\frac{\left(z f^{\prime}(z)\right)^{\prime}}{f^{\prime}(z)}-\frac{1-A}{1-B}\right] & \geq\left(\frac{k}{4}+\frac{1}{2}\right)\left(\frac{1-A r}{1-B r}\right)-\left(\frac{k}{4}-\frac{1}{2}\right)\left(\frac{1+A r}{1+B r}\right)-\frac{1-A}{1-B} \\
& =\frac{(A-B)-\frac{k}{2}(1-B)(A-B) r-B(A-B) r^{2}}{(1-B)\left(1-B^{2} r^{2}\right)}
\end{aligned}
$$

Hence $f \varepsilon C\left(\frac{1-A}{1-B}\right)$ for $|z|<r_{1}, r_{1}$ is given by (3.2).

From Theorem 3.4 and relation (1.4) we have the following:

THEOREM 3.5. Let $f \varepsilon R_{k}[A, B]$. Then $f \varepsilon S^{*}\left(\frac{1-A}{1-B}\right)$ for $|z|<r_{1}$ where $r_{1}$ is given by (3.2).

THEOREM 3.6. Let $\alpha$ and $m$ be any positive integers and $f \varepsilon R_{k}[A, B]$. Then the function $F$ defined by

$$
(F(z))^{\alpha}=\frac{\alpha+m}{z^{m}} \int_{0}^{z} t^{m-1}(f(t))^{\alpha} d t
$$

belongs to $S^{*}\left(\frac{1-A}{1-B}\right)$ for $|z|<r_{1}, r_{1}$ is given by (3.2).

PROOF. Let $J(z)=\int_{0}^{z} t^{m-1}(F(t))^{\alpha} d t$ and so

$$
(F(z))^{\alpha}=\frac{\alpha+m}{z^{m}} J(z)
$$

and

$$
\frac{\alpha z F^{\prime}(z)}{F(z)}=\frac{z J^{\prime}(z)}{J(z)}-m
$$

or

$$
\begin{gathered}
\frac{z F^{\prime}(z)}{F(z)}=\frac{1}{\alpha} \frac{z J^{\prime}(z)-m J(z)}{J(z)}=\frac{N(z)}{D(z)} \\
N(0)=0=D(0)
\end{gathered}
$$


By a result of Bernardi [8] and Theorem 3.5, $D(z)$ is a $(m+\alpha-1)$-valent starlike function for $|z|<r_{1}$. Also

$$
\begin{aligned}
\frac{N^{\prime}(z)}{D^{\prime}(z)} & =\frac{1}{\alpha}\left\{\frac{\left(z J^{\prime}(z)\right)^{\prime}-m J^{\prime}(z)}{J^{\prime}(z)}\right\} \\
& =\frac{z f^{\prime}(z)}{f(z)}
\end{aligned}
$$

Now, by Theorem 3.5, $f \varepsilon S^{*}\left(\frac{1-A}{1-B}\right)$ for $|z|<r_{1}$ and this implies that $\frac{N^{\prime}(z)}{D^{\prime}(z)} \varepsilon P\left(\frac{1-A}{1-B}\right)$ for $|z|<r_{1}$. Hence

$$
\frac{N(z)}{D(z)} \varepsilon P\left(\frac{1-A}{1-B}\right) \text { for }|z|<r_{1} \text {, see [8]. }
$$

This proves our result.

Similarly, we can prove the following:

THEOREM 3.7. Let $\alpha$ and $m$ be positive integers and $f \varepsilon V_{k}[A, B]$. Let $F$ be defined by (3.3). Then $f \varepsilon C\left(\frac{1-A}{1-B}\right)$ for $|z|<r_{1}$ where $r_{1}$ is given by (3.2).

We now prove:

THEOREM 3.8. Let $f$ and $g \varepsilon R_{k}[A, B]$ and, for $\alpha, m$ positive integers, let $F$ be defined as

$$
(F(z))^{\alpha}=\frac{(m+\alpha)}{(g(z))^{m}} \int_{0}^{z} t^{m-1}(f(t))^{\alpha} d t
$$

Then $F \varepsilon S^{*}\left(\frac{1-A}{1-B}\right)$ for $|z|<r_{0}$

where $r_{0}=\min \left(r_{1}, r_{2}\right), r_{1}$ is given by (3.2) and $r_{2}$ is the least positive root of the equation

$$
\left\{(1-B)-\alpha(1-A)-\left\{(A-B(1+2 m)\} r+\{(A-B)+2 m(A-B)+\alpha(1-A)\} r^{2}=0,\right.\right.
$$

PROOF. Let $J_{1}(z)=\frac{\alpha+m}{z^{m}} \int_{0}^{z} t^{m-1}(f(t))^{\alpha} d t$.

Then $(F(z))^{\alpha}=\left(\frac{z}{g(z)}\right)^{m} J_{1}(z)$, where by Theorem 3.6, $J_{1} \varepsilon S^{*}\left(\frac{1-A}{1-B}\right)$ for $|z|<r_{1}$.

So

$$
\frac{\alpha z F^{\prime}(z)}{F(z)}-\frac{z J_{1}^{\prime}(z)}{J_{1}(z)}+m\left(1-\frac{z g^{\prime}(z)}{g(z)}\right)
$$

Thus

$$
\begin{aligned}
\operatorname{Re}\left[\frac{z F^{\prime}(z)}{F(z)}-\frac{1-A}{1-B}\right] \geq \frac{1}{\alpha}\left[\left(1+\frac{B-A}{1-B} r\right) /(1+r)\right]+\left(\left[\frac{2 m}{\alpha}(B-A) r\right] /(1-R)\right)-\frac{1-A}{1-B} \\
\quad=\left\{\frac{\left\{(1-B-\alpha+\alpha A)+[(B-A)(1+2 m)] r+[(A-B)+2 m(A-B)+\alpha(1-A)] r^{2}\right\}}{\alpha(1-B)\left(1-r^{2}\right)}\right\}
\end{aligned}
$$

This implies $\operatorname{Re} \frac{z F^{\prime}(z)}{F(z)} \geq \frac{1-A}{1-B}$ for $|z|<r_{2}$, where $r_{2}$ is the least positive root of (3.5). Hence $F \varepsilon S^{*}\left(\frac{1-A}{1-B}\right)$ for $|z|<r_{0}$, where $r_{0}=\min \left(r_{1}, r_{2}\right)$.

Similarly, we have the following:

THEOREM 3.9. Let $f$ and $g \varepsilon V_{k}[A, B]$ and, for $\alpha, m$ positive integers, let $F$ be defined by (3.4). Then $F \varepsilon C\left(\frac{1-A}{1-B}\right)$ for $|z|<r_{0}$, where $r_{0}$ is as given in Theorem 3.8.

THEOREM 3.10. Let $g \varepsilon V_{k}[A, B]$ and $\frac{f^{\prime}(z)}{8^{\prime}(z)} \varepsilon P[A, B]$ and let $F$ be defined by 


$$
F(z)=\frac{m+1}{z^{m}} \int_{0}^{z} t^{m-1} f(t) d t,
$$

where $m$ is any positive integer. Then there exists a function $G$ such that

$$
\frac{F^{\prime}(z)}{G^{\prime}(z)} \varepsilon P\left(\frac{1-A}{1-B}\right), G \varepsilon C\left(\frac{1-A}{1-B}\right)
$$

for $|z|<r_{1}$, where $r_{1}$ is given by (3.2).

PROOF. Let

$$
G(z)=\frac{m+1}{z^{m}} \int_{0}^{z} t^{m-1} g(t) d t .
$$

Then, by Theorem 3.7 with $\alpha=1, G \varepsilon C\left(\frac{1-A}{1-B}\right)$ for $|z|<r_{1}$ and $r_{1}$ is defined by (3.2). Now

$$
\begin{aligned}
\frac{F^{\prime}(z)}{G^{\prime}(z)} & =\frac{z^{m} f(z)-m\left(\int_{0}^{z} t^{m-1} f(t) d t\right)}{z^{m} q(z)-m\left(\int_{0}^{z} t^{m-1} g(t) d t\right)} \\
& =\frac{\int_{0}^{z} t^{m} f^{\prime}(t) d t}{\int_{0}^{z} t^{m} g^{\prime}(t) d t}=\frac{N(z)}{D(z)}
\end{aligned}
$$

Also

$$
\frac{N^{\prime}(z)}{D^{\prime}(z)}=\frac{f^{\prime}(z)}{g^{\prime}(z)} \varepsilon P[A, B] \text { for }|z|<r_{1}
$$

Thus, by Lemma 2.2, we have $\frac{N(z)}{D(z)} \varepsilon P[A, B] \subset P\left(\frac{1-A}{1-B}\right)$ for $|z|<r_{1}$ and this proves our result.

\section{REFERENCES}

1. JANOWSKI, W. Some extremel problems for certain families of analytic functions, Ann. Polon. Math. 28 (1973), 297-326.

2. ROBERTSON, M. S. On the theory of univalent functions, Ann. Math. 37 (1936), 374-408.

3. KARUNAKARAN, V. and PADMA, K. Functions of bounded radius rotation, Indian J. Pure Appl. Math. 12 (1981), 621-627.

4. PAATERO, V. Uber die Konforme Abbildung von Gebieten deren Rander von beschrankter Drehung sind, Ann. Acad. Sci. Fenn. Ser. A33 (1933), 77.

5. PARVATHAM, R. and SHANMUGAM, T. N. On analytic functions with reference to an integral operator, Bull. Austral. Math. Soc. 28 (1983), 207-215.

6. LIBERA, R. J. Some classes of regular univalent functions, Proc. Amer. Math. Soc 16 (1965), 755-758.

7. ANH, V. V. and TUAN, P. D. Extremal problems for a class of functions of positive real part and applications, J. Aust. Math. Soc. 41 (1986), 152-164.

8. BERNARDI, S. M. Convex and starlike univalent functions, Trans. Amer. Math. Soc. 135 (1969), 429-446. 


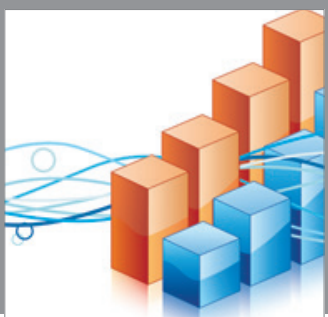

Advances in

Operations Research

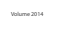

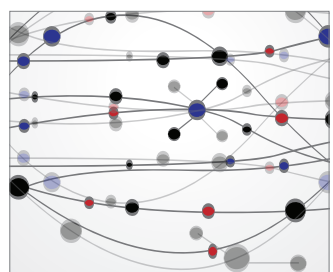

\section{The Scientific} World Journal
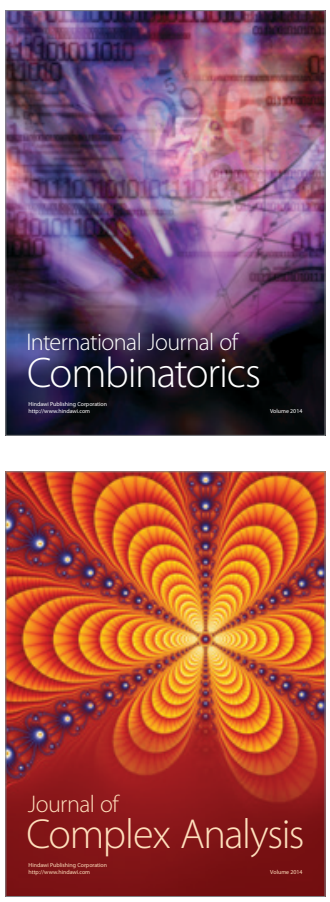

International Journal of

Mathematics and

Mathematical

Sciences
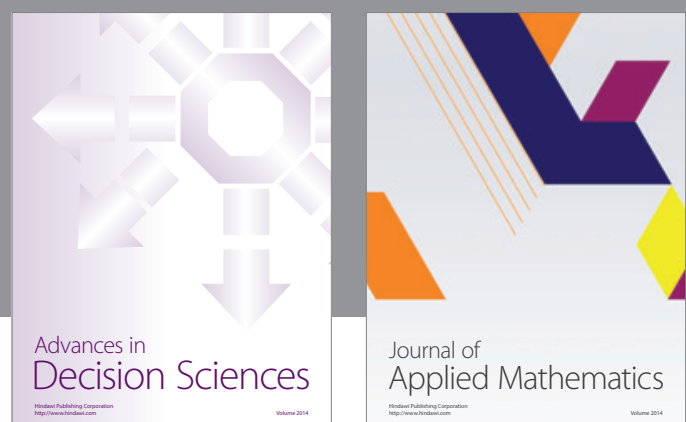

Journal of

Applied Mathematics
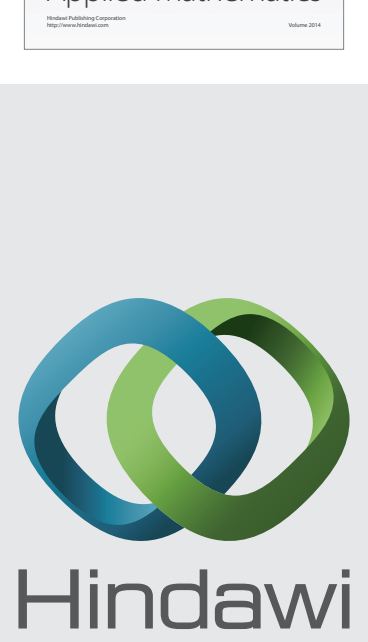

Submit your manuscripts at http://www.hindawi.com
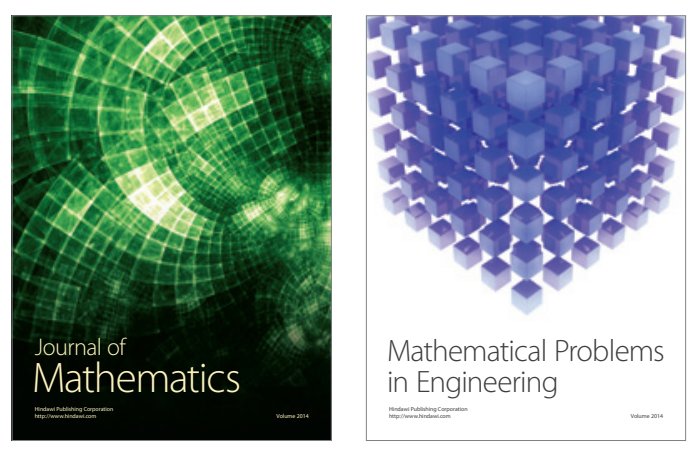

Mathematical Problems in Engineering
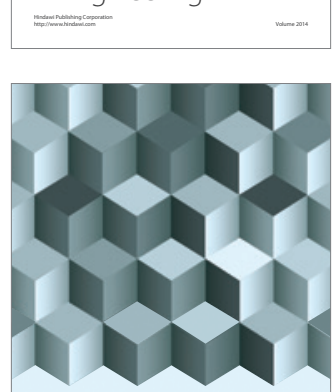

Journal of

Function Spaces
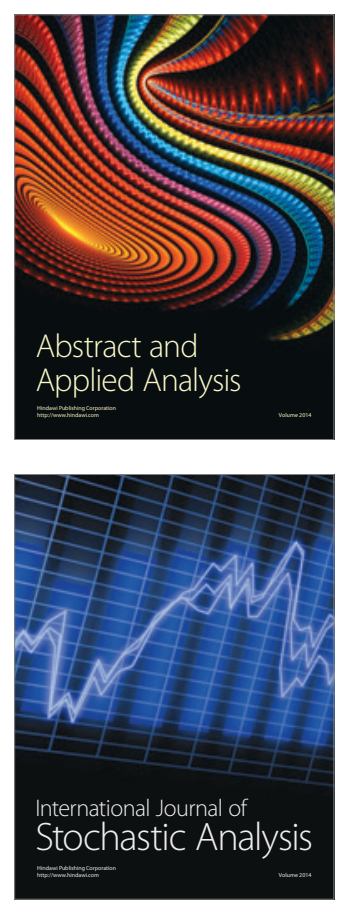

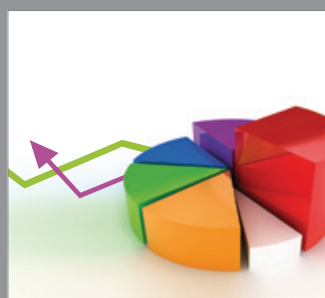

ournal of

Probability and Statistics

Promensencen
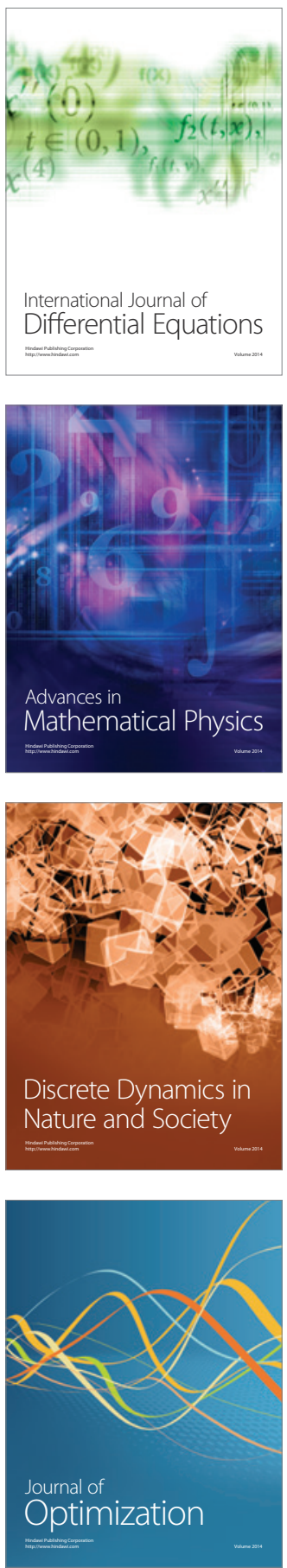